\title{
EFECTO DE LA SUPLEMENTACIÓN CON Moringa oleifera SOBRE EL COMPORTAMIENTO PRODUCTIVO DE OVINOS ALIMENTADOS CON UNA DIETA BASAL DE PASTO GUINEA (Panicum maximun Jacq.)
}

\author{
Nadir Reyes Sánchez ${ }^{1}$, Rosario Rodríguez", Bryan Mendieta Araica1, Lester Mejía Sovalbarro², Ana \\ P. Mora Taylor². \\ ${ }^{1}$ Docentes Investigadores, Facultad de Ciencia Animal, Universidad Nacional Agraria, Managua, Nicaragua. Km 12 1/2 car- \\ retera norte.Apdo.453 Email: nadir.reyes@una.edu.ni \\ ${ }^{2}$ Graduados de la Carrera Ingeniería en Zootecnia, Universidad Nacional Agraria
}

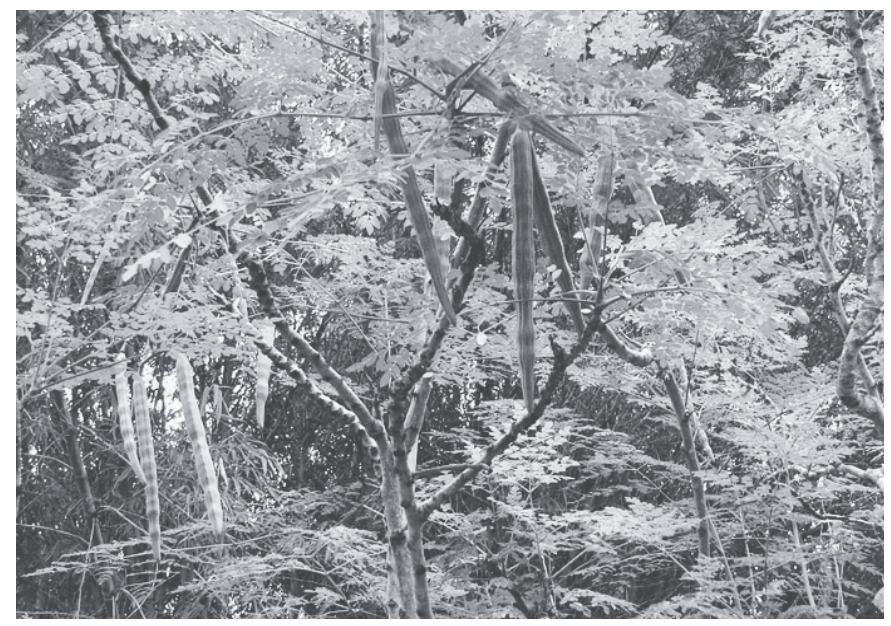

\section{RESUMEN}

El presente estudio se realizó en la finca Santa Rosa propiedad de la Universidad Nacional Agraria, localizada geográficamente en los $12^{\circ} 08^{\prime} 15^{\prime \prime}$ latitud Norte y $86^{\circ} 09^{\prime} 36^{\prime \prime}$ longitud Este, en el Departamento de Managua, Nicaragua con el objetivo de evaluar el comportamiento productivo de ovinos alimentados con una dieta basal de pasto guinea (Panicum máximum Jacq) y suplementados con diferentes niveles de Moringa oleifera. Se utilizaron 18 corderos mestizos (Pelibuey x Black belly) con pesos iníciales promedio de $20 \pm 2 \mathrm{~kg}$, los cuales fueron desparasitados, vitaminados y distribuidos en un Diseño Completamente Aleatorio con tres tratamientos: Panicum máximum ad-libitum, P. máximum ad-libitum $+0.35 \mathrm{~kg}$ MS $M$. oleifera, y $P$. máximum ad-libitum $+0.50 \mathrm{~kg}$ MS M. oleifera. Las variables estudiadas fueron: consumo total de MS (CTMS), ganancia media diaria (GMD) y conversión alimenticia (CA). Se realizó análisis de varianza y comparaciones de medias con la Prueba de Tukey utilizando MINITAB, versión 12.0. Los resultados de los análisis de varianza $(\mathrm{P}<0,05)$ mostraron que el mejor CTMS, GMD y CA se obtiene con la utilización de $P$. máximum ad-libitum $+0.50 \mathrm{~kg}$ MS M. oleifera $(0.8 \mathrm{~kg}$ MS/animal/día, $117.97 \mathrm{~g} / \mathrm{animal} /$ día y 6.78), el que difiere estadísticamente $(\mathrm{P}<0.05)$ del tratamiento con Panicum máximum ad-libitum, (0.57 kg MS/animal/día, $30.85 \mathrm{~g} /$ animal/día y 18.47$)$ pero $(\mathrm{P}>0.05)$ del tratamiento $P$. máximum
ABSTRACT

An experiment was conducted at Santa Rosa farm, National University of Agriculture in Managua, Nicaragua, located geographically at $12^{\circ} 08^{\prime} 15^{\prime \prime}$ north and $86^{\circ} 09^{\prime} 36^{\prime \prime}$ east, in order to evaluate the effect of supplementation with different levels of Moringa oleifera forage on productive behavior of sheep feeding with a basal diet of Panicum maximum Jacq. Eighteen tropical cross breed lambs with an average initial body weight of $20 \pm 2 \mathrm{~kg}$ were used in a Completely Randomized Design and with three treatments: Panicum máximum ad-libitum, $P$. máximum ad-libitum $+0.35 \mathrm{~kg}$ DM $M$. oleifera and $P$. máximum ad-libitum $+0.50 \mathrm{~kg}$ DM M. oleifera. Before the start up of the trial, all animals were injected with Vitamin $\mathrm{AD}_{3} \mathrm{E}$ and were treated against external and internal parasites. Data were analyzed through analysis of variance using the Minitab Statistical Software Version 12.0 (Minitab, 1998). Tukey's pairwise comparison procedure was used when the difference between treatments means was significant. Supplementation with Moringa increased $(\mathrm{P}<0.05)$ Total DM intake from 0.57 to 0.73 and $0.80 \mathrm{~kg}$ DM day- 1 , average daily weight gain from 30.9 to 90.9 and $118.0 \mathrm{~g}$ day- 1 and feed conversion ratio from 18.5 to 8.0 and 6.8 for $P$. maximum only and supplementation with $0.35 \mathrm{~kg}$ and $0.50 \mathrm{~kg}$ DM of Moringa, respectively. The results showed that the inclusion of Moringa as a protein supplement for sheep fed with low quality diets improved Total DM intake, average daily weight gain and feed conversion ratio. 
$90.91 \mathrm{~g} /$ animal/día y 8.02). En conclusión el forraje de $M$. oleifera como suplemento proteico para ovinos consumiendo una dieta basal de $P$. máximum incrementa la ganancia de peso y mejora el consumo total de MS y la conversión alimenticia. Palabras clave: Marango, ganancia media diaria, consumo animal, conversión alimenticia.

Abreviaturas: MS, materia seca, CTMS: consumo total de MS, GMD, ganancia media diaria
Keywords: Marango, average daily weight gain, intake, feed efficiency.
A nivel de economía campesina la crianza de ovinos tiene ventajas comparativas, tales como: necesitan poca inversión financiera, el riesgo de la inversión se puede repartir entre varios animales, son más fáciles de manejar y controlar, tienen alto potencial reproductivo, requieren menores cantidades de alimento y se pueden sacrificar para autoconsumo. Adicionalmente, su carácter de rumiante y de especie adaptada a las zonas tropicales le otorga ventajas adicionales, como la capacidad de utilizar alimentos con alto contenido de fibra que no son aptos para la alimentación de monogástricos y de ésta forma, pueden contribuir apropiadamente a un eficaz uso de los recursos fibrosos disponibles en la economía campesinos.

La producción de pastos y forrajes esta sometida a variaciones pluviométricas que se traducen en problemas de baja disponibilidad y baja calidad durante todo el año, pero principalmente en la época de seca, originando una disminución en la eficiencia de producción de los rebaños.

Una estrategia potencial de los pequeños y medianos productores, para incrementar la disponibilidad y calidad de los alimentos para rumiantes puede ser a través de la utilización de árboles y arbustos forrajeros (Pezo, 1991) que tienen gran potencial para mejorar los sistemas de producción animal. Estos materiales poseen alto rendimiento de forraje, pueden tolerar mejor el mal manejo y tienen capacidad de rebrotar y ofrecer forraje de buena calidad en localidades de sequía prolongada (Perdomo, 1991). La mayoría de estas especies son de fácil propagación y no requieren de tecnología avanzada, ni de gran cantidad de insumos externos.

El marango (Moringa oleifera Lam) es uno de estos árboles forrajeros que crece bien en todo tipo de suelos desde ácidos hasta alcalinos (Duke, 1983), su producción de forraje es entre 24 y 99 ton MS/ha/año, las hojas frescas contienen entre 17 y $24.6 \%$ de PB, 2.73 Mcal de EM/kg MS (Makkar y Becker, 1996; Makkar y Becker, 1997; Foild et al., 1999; Aregheore, 2002, Reyes et al 2006) es rico en vitaminas A, B y C, calcio, hierro y en dos aminoácidos esenciales (metionina y cistina) generalmente deficientes en otros alimentos (Makkar y Becker, 1996).

El objetivo del presente trabajo de investigación fue evaluar el comportamiento productivo (consumo diario de alimento, ganancia media diaria y conversión alimenticia) de ovinos alimentados con una dieta basal de pasto guinea (Panicum máximum Jacq) y suplementados con diferentes niveles de Moringa oleifera.

\section{MATERIALES Y MÉTODOS}

Localización del área experimental. El presente estudio se realizó en la granja ovina, Finca Santa Rosa, propiedad de la Universidad Nacional Agraria, Managua, Nicaragua, localizada geográficamente a los $12^{\circ} 08^{\prime} 15^{\prime \prime}$ latitud Norte y los $86^{\circ} 09^{\prime} 36^{\prime \prime}$ 'longitud Este, a una altitud de $56 \mathrm{msnm}$. Las condiciones climáticas en el sitio experimental corresponden a una zona de vida ecológica de bosque tropical seco, con un rango de precipitación histórica de $1403 \mathrm{~mm}$, humedad relativa de 72\% y una temperatura media anual de $27.3{ }^{\circ} \mathrm{C}$. El régimen pluviométrico de la zona se caracteriza por presentar una época seca prolongada entre los meses de Noviembre a Abril y una temporada húmeda entre los meses de Mayo a Octubre, durante la realización del presente trabajo investigativo la precipitación fue de $1198 \mathrm{~mm}$.

Manejo de las áreas de pasto guinea y marango. Para la suplementación de los animales se utilizó forraje de un área establecida con Marango en el año 2000. El Marango lo establecieron como cultivo puro con semilla botánica a una distancia de 5 centímetros entre plantas y 40 centímetros entre surcos, sin riego, ni fertilización. Las semillas fueron plantadas a $1 \mathrm{~cm}$ de profundidad, colocando dos semillas por golpe. Durante el experimento se hicieron dos chapias manuales para mantener el cultivo libre de malezas. Antes de iniciar el experimento se realizó un corte de uniformidad para asegurar la disponibilidad de un rebrote de aproximadamente 45 días de edad.

Un área de pasto guinea sin riego, ni fertilización fue utilizada para la producción del forraje de la dieta basal de los corderos. El corte del pasto se realizaba diariamente 
con machete y se picaba mecánicamente. El marango se cosechaba diariamente a una altura de $25 \mathrm{~cm}$ usando machete, y se picaba en pedazos de aproximadamente 2 $\mathrm{cm}$ de longitud, usando una picadora mecánica. Antes de picarlo se separaban los tallos que tuvieran un diámetro mayor a los cinco mm para asegurar una composición uniforme del forraje suministrado. El pasto guinea y el forraje de Marango fueron ofertados en comederos separados, dos veces al día, en la mañana a las 8:00 am y por la tarde a las 15:00 pm, respectivamente. La cantidad total de pasto guinea ofertado ( $\mathrm{kg} \mathrm{MS/animal/día)} \mathrm{fue} \mathrm{el}$ mismo para los tres tratamientos en estudio.

Manejo y alimentación de los animales. Para el experimento se utilizaron 18 corderos mestizos (Pelibuey x Black belly) del hato de la Universidad Nacional Agraria, con un peso corporal inicial de $20 \pm 2 \mathrm{~kg}$ y cinco meses de edad aproximadamente. Antes de iniciar el ensayo a los animales se les aplicó vitaminas $\mathrm{AD}_{3} \mathrm{E}$ y fueron tratados contra parásitos externos e internos. A todos los animales se les suministró agua ad libitum. Los animales fueron pesados al inicio del ensayo y alojados en cubículos techados y bien ventilados, luego se pesaban cada 15 días, antes de suministrarles alimento, para determinar la ganancia de peso.

El consumo voluntario de los alimentos (pasto guinea y Marango, separadamente) fue estimado cada 15 días durante todo el período experimental por el método convencional, calculando la diferencia entre el alimento ofrecido y el alimentado rechazado. Por ello, los alimentos se pesaban antes de suministrárselos a los animales y al día siguiente antes de ofrecer el nuevo alimento, el alimento rechazado era colectado y pesado por separado.

El pasto guinea y el forraje de Marango ofrecido se muestreó cada 15 días y se almacenaron en un congelador. Las muestras posteriormente fueron secadas en un horno de circulación forzada de aire a $65^{\circ} \mathrm{C}$ por 48 horas, luego se molieron hasta obtener un tamaño de partícula de $1 \mathrm{~mm}$, y se almacenaron en recipientes de vidrios debidamente identificados, para subsecuentes análisis químicos.

Análisis químicos. A las muestras de pasto guinea y marango se les determinó materia seca y proteína bruta. La MS fue determinada por secado de la muestra en un horno a $105^{\circ} \mathrm{C}$ durante 6 horas. El Nitrógeno total (N) fue determinado por el procedimiento de Kjeldhal (Kass y Rodríguez, 1993) y el contenido de proteína bruta fue calculado mediante la fórmula $\mathrm{PB}=\mathrm{N}$ x 6.25 según los métodos oficiales de la Association of Official Analytical Chemists (AOAC, 1990).
Análisis financiero . Con la finalidad de comparar los costos por tratamiento así como determinar el beneficio económico que habrá al sustituir uno de los tratamientos por otro, se realizó un análisis de presupuestos parciales con la metodología sugerida por Mendieta (1996). Los presupuestos parciales para cada tratamiento se basaron en los costos debidos al alimento. En general se consideran cuatro partidas básicas que se clasifican como sigue: Nuevas entradas: A) Costos reducidos (del rubro que se piensa sustituir) y B) Nuevos ingresos (del rubro que se piensa introducir); Nuevas salidas: C) Nuevos costos (del rubro que se piensa introducir) y D) Ingresos reducidos (del rubro que se piensa sustituir). Las diferencias entre las nuevas entradas $(\mathrm{A}+\mathrm{B})$ y las nuevas salidas $(\mathrm{C}+\mathrm{D})$ indica si el cambio produce utilidades. La utilidad se calculó mediante la siguiente fórmula $\mathrm{U}=(\mathrm{A}$ $+\mathrm{B})-(\mathrm{C}+\mathrm{D})$, si este resultado fuese negativo o muy pequeño el cambio no se justifica.

Diseño experimental y análisis estadístico. Para este trabajo se utilizó un Diseño completamente al azar (DCA), con tres tratamientos y 6 repeticiones por tratamiento. Se utilizaron 18 corderos que se distribuyeron aleatoriamente quedando 6 animales por tratamiento. Los tratamientos evaluados fueron: T1 Panicum máximum ad-libitum; T2: P. máximum ad-libitum $+0.35 \mathrm{~kg}$ MS Moringa oleifera y T3: $P$. máximum ad-libitum $+0.50 \mathrm{~kg}$ MS $M$. oleifera.

Las variables estudiadas en el experimento fueron las siguientes: Ganancia de peso (GP): aumento de peso que experimentan los animales durante todo el ensayo, se calcula mediante la siguiente fórmula: $\mathrm{GP}=$ Peso final (PF) - Peso inicial (PI); Ganancia Media Diaria (GMD): es un índice que representa las unidades de peso vivo que aumenta un animal cada día y generalmente se evalúa en kilogramos por día, los días corresponden al periodo de intervalo entre el pesaje inicial y el pesaje final, se calcula mediante la siguiente fórmula: $\mathrm{GMD}=(\mathrm{PF}-\mathrm{PI}) /$ Duración del período experimental (días); Consumo de Alimento: fue estimado por el método convencional: Consumo $=($ alimento suministrado alimento rechazado) y la Conversión Alimenticia (CA) que es un índice que representa los $\mathrm{kg}$ de alimento que debe consumir un animal para aumentar un $\mathrm{kg}$ de peso vivo y fue estimada mediante la siguiente fórmula: $\mathrm{CA}=$ consumo diario de alimento $(\mathrm{kg}) / \mathrm{GMD}(\mathrm{kg})$

Análisis estadístico. Se realizó Análisis de varianza para determinar el efecto de los tratamientos sobre las variables en estudio utilizando el General Lineal Model del MINITAB Statistical Software versión 
12.0 para computadoras personales (Minitab, 1998). Las comparaciones de medias se realizaron por el procedimiento de Tukey cuando las diferencias entre los medias eran significativas $(\mathrm{P}<0.05)$.

\section{RESULTADOS Y DISCUSIÓN}

El valor nutritivo de las plantas forrajeras esta en función de la composición química y del consumo voluntario de los animales. Los contenidos de materia seca y proteína bruta de los alimentos utilizados en el presente experimento fueron determinados en el Laboratorio de Suelos y Agua de la Universidad Nacional Agraria y se muestran en la Tabla 1.

El pasto $P$. máximum utilizado en este experimento fue el típico forraje utilizado en la época de seca con bajo contenido de proteína bruta. Leng (1990) define forraje de baja calidad como aquel forraje que tiene menos del $8 \%$ de PB en base seca y sugiere que la suplementación con apropiados niveles de nutrientes permite alcanzar altos niveles de producción animal. Estudios nutricionales han mostrado que el uso de arboles y arbustos forrajeros como suplementos proteicos aumentan el valor nutritivo de alimentos fibrosos de baja calidad (Goodchild and McMeniman 1994; Ibrahim et al., 2001).

Los contenido de materia seca (\%) y proteina bruta (\%) de los alimentos utilizados en el experimento fueron $18.6(0.18)$ y $6.2(0.52)$ para Panicum maximum y 15.4 (0.28) y $18(0.70)$ para Moringa oleifera respectivamente (desviación estándar entre paréntesis) La canti- dad de forraje consumido es una de las principales determinantes de la producción animal en sistemas basados en alimentación con pastos y forrajes. El apetito de los rumiantes no es constante y varía según el tipo de alimento. El consumo voluntario de alimento es el principal factor que controla el consumo diario de nutrientes y el aumento en la ganancia de peso de los animales.

El segundo factor que incide sobre la producción animal es la concentración de nutrientes de los alimentos. Los alimentos voluminosos, de baja calidad, tienen baja concentración de PB y alta concentración de fibra, lo que limita el consumo de MS, mas por la capacidad física del rumen que por mecanismos fisiológicos o digestibilidad (Minson et al., 1993). Cuando el contenido de PB en los pastos es menor del 7\% las bacterias no pueden digerir rápidamente las fibras y el material es retenido por un mayor tiempo en el rumen del animal.

Consumo de materia seca total. Este estudio muestra que el Consumo de Materia Seca Total consistentemente incrementa $(\mathrm{P}<0.05)$ en la medida que el nivel de suplementación con marango aumenta y fue significativamente mayor $(\mathrm{P}<0.05)$ para los animales suplementados con $0.5 \mathrm{~kg}$ MS M. oleifera (Figura 1). Los animales suplementados con 0.5 y $0.3 \mathrm{~kg}$ MS de forraje de marango incrementan $(\mathrm{P}<0.05)$ el consumo diario total en 0.23 y $0.16 \mathrm{~kg} \mathrm{MS}$, respectivamente, comparados con los animales alimentados con la dieta basal de $P$. máximum.

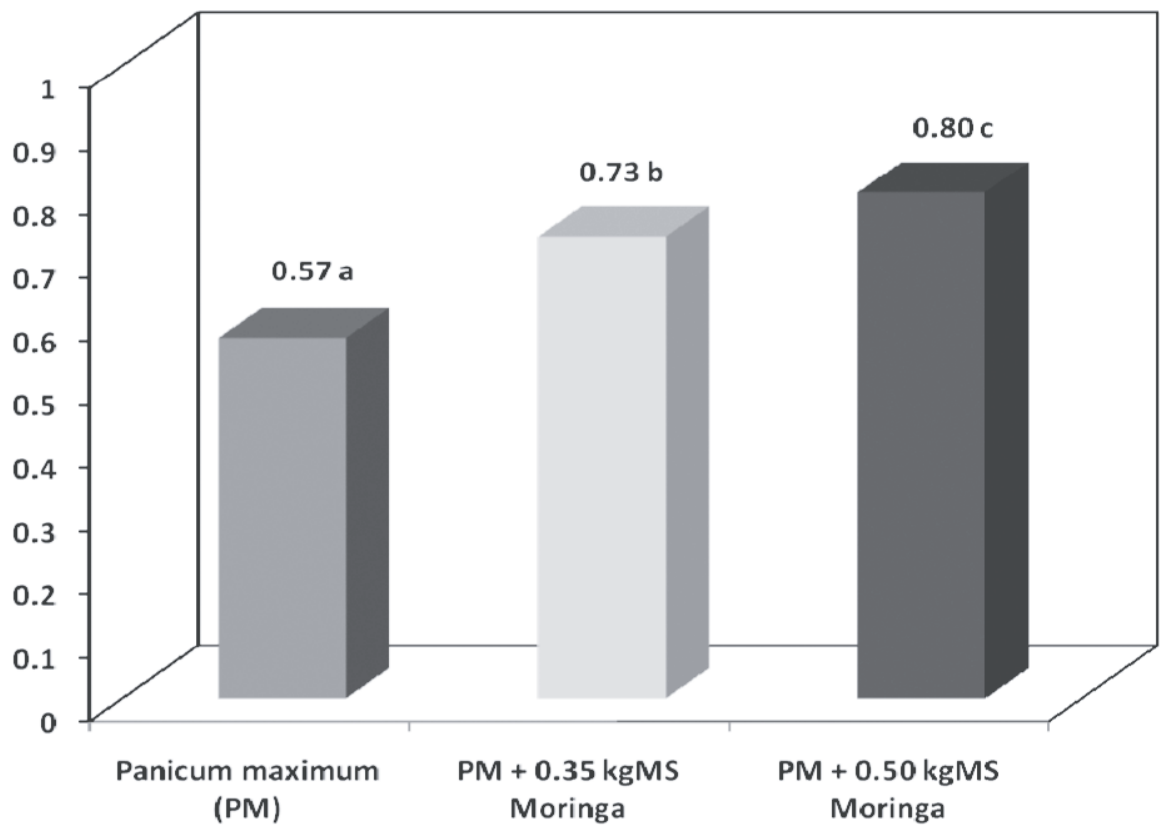

Figura 1. Efecto de la suplementación con $M$. oleífera sobre el consumo de materia seca total (kg MS/animal/día) de ovinos alimentados con una dieta basal de pasto guinea (Panicum maximun jacq.). 
Estos resultados son superiores a los reportados por Ríos et al (2005) que encontró valores entre 0.38 y 0.48 $\mathrm{kg} \mathrm{MS} /$ animal/día para corderos consumiendo una dieta basal de Pennisetum purpureum ofrecido ad libitum y suplementados con forraje de Morus sp (23.1\% PB) y Gliricidia sepium (24.4\% PB), respectivamente. Por otro lado, son similares a los reportados por Medina y Sánchez (2006) quienes observaron consumos entre 0.54 y $0.73 \mathrm{~kg} \mathrm{MS} /$ animal/día para corderos consumiendo una dieta basal de Pennisetun purpureum Schum suplementados o no con follaje de L. leucocephala (19\% PB).

De acuerdo con Malafaia et al. (2003) cuando el contenido de PB de la ración es menor al 7\% de PB en base seca, el consumo de materia seca se reduce debido a deficiencias en el suministro de nitrógeno para los microorganismos del rumen. El menor consumo en los animales que solo consumieron pasto $P$. máximum fue probablemente debido al bajo contenido de $\mathrm{PB}$ de la ración, que estuvo por debajo del nivel critico requerido para una eficiente actividad microbial.

Los resultados muestran que la alimentación de ovinos con forraje de marango como suplemento proteico a una dieta basal con forraje de baja calidad $(P$. máximum) resulta en un aumento altamente significativo en el consumo de MS. Esto coincide con lo reportado por Castellón y Gonzáles (1996), quienes indican que la suplementación con forraje de marango a animales que consumían heno de pasto estrella incrementaba significativamente el consumo de materia seca total.

La tendencia de aumento del consumo total de materia seca cuando se incluye mayor cantidad de Marango en la ración, podría explicarse debido a que los componentes del contenido celular del Marango tienen altos niveles de sustancias liberadoras de energía, que son conocidas por incrementar la síntesis de proteína microbiana, la que es desdoblada y utilizada por el animal (Becker, 1995). Esto es también coincidente con lo planteado por Googchild y McMeniman (1994) quienes indican que la inclusión de $20-50 \%$ de plantas ricas en proteínas en la dieta, da lugar a un aumento del consumo total entre 10 y $45 \%$ debido a que se mejora la actividad microbiana, como resultado del aumento de nutrientes esenciales disponibles para los microorganismos del rumen. Por otro lado, Becker (1995) observó que las tasas de fermentación de raciones con diferentes proporciones de Marango producen variaciones en la fermentación en comparación cuando ambos alimentos se suministraban por separado. Esto demuestra que existe evidencia del efecto asociativo en la digestión y asimilación de ambos alimentos, cuando son suministrados al mismo tiempo.

Ganancia media diaria. Para condiciones tropicales, la cría de ovinos es una actividad complementaria a la producción de bovinos o una producción de subsistencia con pobres niveles productivos (GMD de 40 a $70 \mathrm{~g}$ ) al manejarse exclusivamente en pastoreo. Sin embargo, se logran aumentos importantes cuando se maneja suplementación en pastoreo (GMD de 120 a 160 g) con un impacto económico importante. Según Vélez (1993) las ganancias de peso en el trópico con razas de pelo son menores y muestran mayor variación, por lo general de 50 - $150 \mathrm{~g} /$ día.

La GMD (g/animal/día) de los animales incrementan significativamente $(\mathrm{P}<0.05)$ en la medida que se incremento el nivel de suplementación con marango (Figura 2). Con el nivel de suplementación de $0.50 \mathrm{~kg}$ MS de Marango/animal/día se obtuvo la mayor $(\mathrm{P}<0.05) \mathrm{GMD}$ aunque no hubo diferencia significativa con el nivel de suplementación de $0.35 \mathrm{~kg}$ MS de Marango/animal/día. La necesidad de energía adicional para eliminar el nitrógeno consumido en exceso podría explicar la ganancia de peso similar obtenida en ambos tratamientos. Por otro lado, la mayor GMD obtenida presumimos sea debido al aporte nutritivo de la suplementación con follaje M. oleifera que pone de manifiesto el mejor balance nutricional presente en las raciones que consumieron los animales suplementados.

Los datos de GMD aquí presentados coinciden con los de Espinoza et al. (2001), Mendoza et al. (2001) y Clavero et al. (1995), que reportan GMD entre 85 y 116 gr/animal/día; pero inferiores a los reportados por Palma y Huerta (1999) que obtuvieron GMD de 170, 172 y 175 g/animal/día para corderos alimentados con 0,10 y $20 \%$ de inclusión de follaje de $L$. leucocephala en la ración. Los resultados obtenidos son superiores a las indicados por Benavides (1986), Díaz et al (1995) y Ríos et al (2005). Benavides (1986), trabajando con niveles de $0,5,10$ y $15 \%$ de inclusión de follaje de morera obtuvo GMD de 60, 75, 85, $101 \mathrm{~g}$ /animal/día, respectivamente. Díaz et al (1995), mencionan GMD de 49.6, 63.3 y 69.7 $\mathrm{g} / \mathrm{animal} /$ día para animales alimentados con una dieta basal de Pennisetum purpureum o suplementados con Pachecoa o Gliricidia respectivamente y Ríos et al (2005), encontraron 54, 56 y 87 gr de GMD para animales alimentados con una dieta basal de Pennisetum purpureum o suplementados con Morus alba o Gliricidia sepium. 


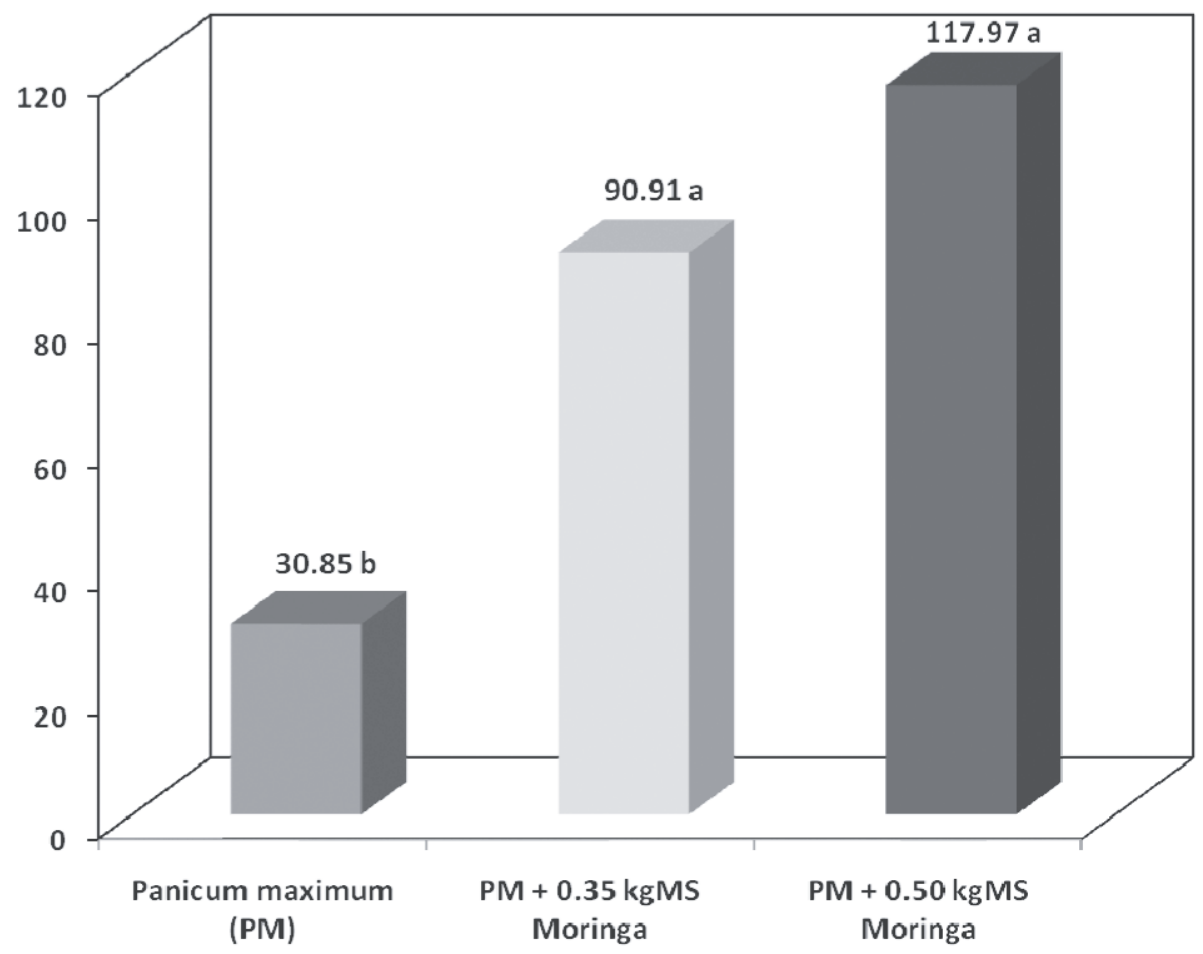

Figura 2. Efecto de la suplementación con M. oleífera sobre la ganancia de peso (g/ animal/día) de ovinos alimentados con una dieta basal de pasto guinea (Panicum maximun jacq.).

Estos resultados pueden ser explicados por que las plantas que incrementan el nivel de proteína en la ración, como el Marango, estimulan un aumento en el nivel de eficiencia en la utilización de la energía metabolizable, producida por una mayor actividad microbial, ejerciendo un efecto positivo sobre la GMD (Ketelaars et al., 1991). Por otro lado, Minson y Milford (1967) reportan que la respuesta a la suplementacion, con plantas de alto contenido proteico, es significativa cuando la proporción de las mismas en la dieta es mayor del 10\%. Dos hipótesis se han propuesto para explicar esta respuesta, uno sugiere que el substrato adicional (amoniaco, péptidos, aminoácidos) aumente la actividad bacteriana en el rumen (Garza et al 1991) y la otra indica que las proteínas dietéticas tienen un efecto sobre la motilidad ruminal y la tasa de pasaje de los alimentos (Kil \& Fruetschel, 1994).

Comportamiento del peso vivo de los animales durante el experimento. La ganancia de peso vivo es la respuesta de los animales ante el consumo de una ración y refleja directamente la cantidad de nutrientes que tuvo disponible durante un periodo de tiempo determinado. Mientras mayor sea la cantidad de nutrientes que un animal tenga disponible y pueda digerir y absorber, mayor será la magnitud del peso que demuestre.
La Figura 3, muestra como los animales suplementados con 0.35 y $050 \mathrm{~kg}$ de MS de Moringa oleífera presentaron un incremento de peso por quincena constante con tendencia lineal positiva durante el transcurso del experimento, en tanto los sometidos a la ración basal de $P$. máximum sin suplementación tuvieron poca fluctuación en la ganancia de peso. Esta situación pudiera estar relacionada con el mayor aporte nutricional principalmente proteína del follaje de $M$. oleifera que recibieron los animales suplementados.

Al respecto, también podría enunciarse que la superioridad de los animales suplementados con 0.35 ó $0.50 \mathrm{~kg}$ MS de Moringa/animal/día, en cuanto a peso vivo puede deberse a un mejor aprovechamiento del nitrógeno proveniente del suplemento forrajero por las bacterias ruminales para la posterior síntesis de los aminoácidos necesarios para su crecimiento. Los ovinos que consumieron únicamente la ración basal de Panicum máximum tienen la menor ganancia de peso debido a que la ración suministrada no proporciona los principios nutritivos necesarios que permitan un adecuado crecimiento y desarrollo de los animales, indicando la necesidad de suplementar los animales.

Conversión alimenticia (CA). Conversión alimenticia se define como la relación entre cantidad de alimento 


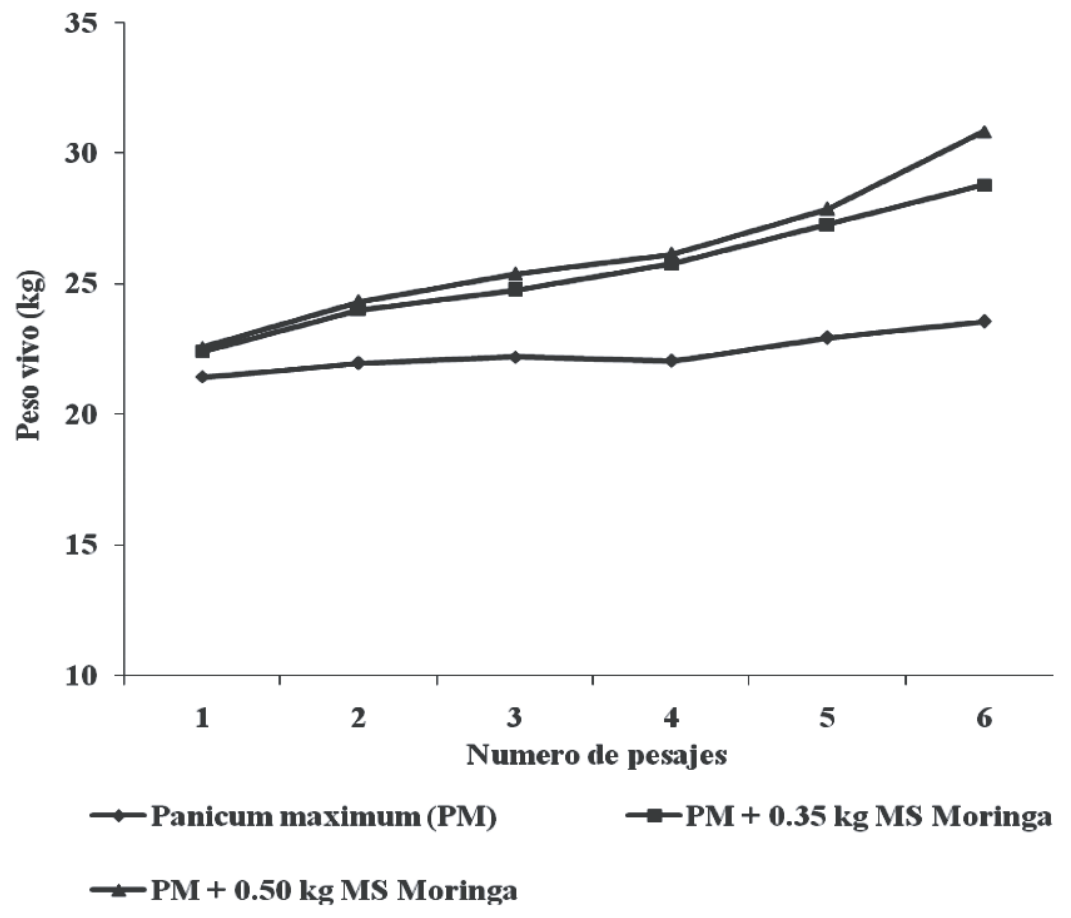

Figura 3. Efecto de la suplementación con M. oleifera sobre la evolución del peso vivo $(\mathrm{kg})$ promedio quincenal de ovinos alimentados con una dieta basal de pasto guinea (Panicum maximun jacq.).

consumido y la ganancia de peso vivo logrado durante un periodo de prueba, lo que incluye la totalidad de alimentos consumidos independientemente sea utilizado para el mantenimiento o crecimiento de los tejidos (Asociación Argentina de Criadores, 1996).

La Conversión Alimenticia de los animales incrementa significativamente $(\mathrm{P}<0.05)$ en la medida que

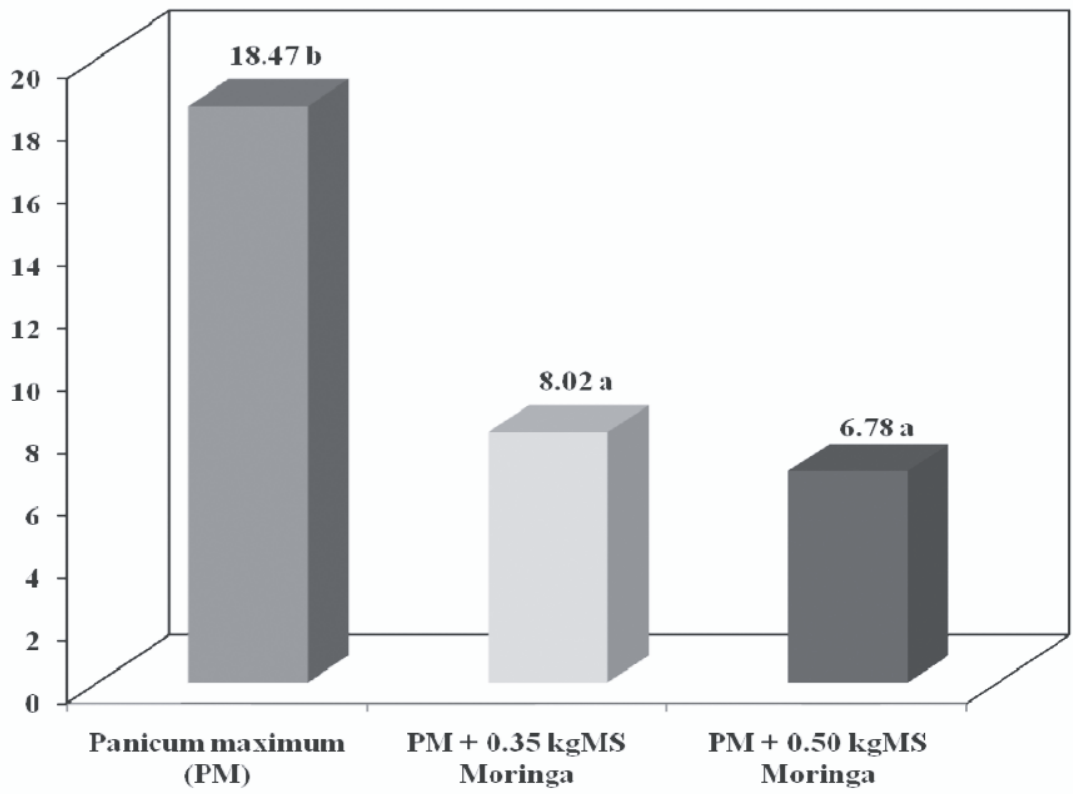

Figura 4. Efecto de la suplementación con M. oleifera sobre la conversión alimenticia de ovinos alimentados con una dieta basal de pasto guinea (Panicum maximun jacq.). 
se incrementó el nivel de suplementación con marango (Figura 4). Con el nivel de suplementación de $0.50 \mathrm{~kg}$ MS de Marango/animal/día se obtuvo la mejor $(\mathrm{P}<0.05)$ conversión alimenticia (6.78) aunque no hubo diferencia significativa con el nivel de suplementación de $0.35 \mathrm{~kg}$ MS de Marango/animal/día (8.02). La suplementación con forraje de M. oleifera parece ejercer su principal efecto en un aumento del consumo voluntario y como resultado una mayor ganancia de peso vivo, mejorando por ende la conversión alimenticia.

La conversión alimenticia de los animales suplementados con $0.5 \mathrm{~kg}$ MS de $M$. oleifera fue mejor que las CA reportadas por Díaz et al. (1995) de 12.4, 9.4 y 8.7, para animales alimentados con una dieta basal de Pennisetum purpureum o suplementados con Pachecoa o Gliricidia respectivamente. El valor de CA obtenido para el tratamiento con $0.5 \mathrm{~kg}$ MS de $M$. oleifera es similar a los valores reportados por Palma y Huerta (1999) de $6.32,6.27$ y 6.22 para corderos alimentados con 0,10 y $20 \%$ de inclusión de follaje de L. leucocephala en la ración, respectivamente.

Podemos concluir que los resultados obtenidos en el presente experimento para conversión alimenticia fueron altamente satisfactorios para ovinos en crecimiento alimentados con $P$. máximum y suplementados con follaje de $M$. oleifera.

Análisis financiero. Utilizando la metodología de presupuestos parciales y la conversión alimenticia como variable de medición (Tabla 2) se demuestra que el tratamiento con mayor inclusión de Marango $(0.5 \mathrm{~kg} M$. oleifera) mantiene el mejor rendimiento financiero con una utilidad bruta de US\$ 13.3 contra US\$ 1.9 obtenidos solo con el suministro de pasto $(0.35 \mathrm{~kg} M$. oleífera $)$.

CA conversión alimenticia, GPVT Ganancia peso vivo total por animal durante el experimento, CTA Consumo Total de Alimento por animal durante el experimento (CA x GPVT), CAL Costo del kg de alimento, CTALC Costo total del alimento consumido durante el experimento (CTA x CAL), PPV Precio del kilogramo de ovino en pie, IT Ingreso total (GPVT x PPV), U utilidad (IT - CTALC).

\section{CONCLUSIONES}

El consumo total de materia seca, ganancia media diaria y conversión alimenticia de ovinos alimentados con una dieta basal de $P$. máximum, se incrementó significativamente en la medida que se incrementó el nivel de suplementación con Moringa. En general, los ovinos suplementados con $0.50 \mathrm{~kg}$ MS de Moringa/ animal/día mostraron la mejor respuesta animal en términos de CTMS, GMD y CA con valores de $0.80 \mathrm{~kg} \mathrm{MS} /$ animal/día, $117.97 \mathrm{~g} / \mathrm{animal} /$ día y 6.78 , respectivamente, con un aumento significativo de la utilidad neta. La suplementación con forraje de Moringa oleífera es una eficiente vía para mejorar la utilización de dietas basales de baja a mediana calidad. La viabilidad financiera de la utilización de Moringa en la alimentación de ovinos, refuerza la recomendación de utilizar el nivel más elevado por ser un recurso altamente disponible y además sumamente rentable, para pequeños productores.

Tabla 2. Análisis financiero utilizando la metodología de presupuestos parciales

\begin{tabular}{lrrllllrr}
\hline Tratamiento & \multicolumn{1}{c}{$\begin{array}{l}\text { CA } \\
(\mathrm{kg})\end{array}$} & GPVT & $\begin{array}{l}\text { CTA (kg) } \\
\text { (US\$) }\end{array}$ & $\begin{array}{l}\text { CAL } \\
\text { (US\$) }\end{array}$ & $\begin{array}{l}\text { CTALC } \\
\text { (US\$) }\end{array}$ & PPVK & IT (US\$) & U (US\$) \\
\hline I & 18.47 & 3.7 & 68.34 & 0.035 & 2.39 & 1.16 & 4.29 & 1.90 \\
II & 8.02 & 10.9 & 87.42 & 0.033 & 2.88 & 1.16 & 12.64 & 9.76 \\
III & 6.78 & 14.2 & 96.28 & 0.033 & 3.18 & 1.16 & 16.47 & 13.29
\end{tabular}

CA conversión alimenticia, GPVT Ganancia peso vivo total por animal durante el experimento, CTA Consumo Total de Alimento por animal durante el experimento (CA x GPVT), CAL Costo del $\mathrm{kg}$ de alimento, CTALC Costo total del alimento consumido durante el experimento (CTA x CAL), PPV Precio del kilogramo de ovino en pie, IT Ingreso total (GPVT x PPV), U utilidad (IT - CTALC) 


\section{REFERENCIAS BIBLIOGRÁFICAS}

AOAC. 1990. Official Methods of Analysis, 15th edition. Association of Official Analytical Chemists, Arlington, VA. $1298 \mathrm{p}$.

Aregheore Em. 2002. Intake and digestibility of Moringa oleifera-batiki grass mixtures for growing goats. Small Rum. Res. 46: 23-28.

ASOCIACIÓN CRIADORES DE ARGENTINA 1996. Disponible en línea: www.imperiorural.com.ar/imperio/ (SEGUIDO Y COLOR NEGRO) estructura/miriam\%20archivos/Bovinos/eficienciaconversionalimenticia. htm

Becker, K. 1995. Studies on utilization of Moringa oleifera leaves as animal feed. Institute for Animal Production in the Tropics and Subtropics, vol. 480. University of Hohenheim, Stuttgart. 15 P.

Benavides E, J. 1986. Efecto de diferentes niveles de suplementación con follaje de morera (Morus sp.) sobre el crecimiento y consumo de cordero alimentados con pastos(Penisetum purpureum) CATIE, Turrialba. Costa Rica.

Castellón, CV; González C, JR. 1996. Utilización del marango (Moringa oleifera) en la alimentación de novillos en crecimiento bajo régimen de estabulación. Tesis Lic. Zootecnia. Managua, Nicaragua, UCA. 44 P.

Clavero, T; Muller, A; Razz, R. 1995. Comportamiento de ovinos suplementados con Leucaena leucocephala. Rev. Arg. Prod. Animal, 15: 413-414.

Díaz, Y; Escobar, A; Viera, J. 1995. Efecto de la substitución parcial del suplemento convencional por follaje de pachecoa (Pachecoa venezuelensis) o gliricidia (Gliricidia sepium) en la alimentacion de corderos postdestete. Livestock Research for Rural Development. Vol 7:1.

Duke J, A. 1983. Handbook of energy crops (Moringa oleifera). Center for new crops and plant products. Purdue University, Indiana, US. http://www.hort.purdue. edu/newcrop/duke_energy/Moringa_oleifera.html.

Espinoza, F; Araque, C; León, L; Quintana, H; Perdomo, E. 2001. Efecto del banco de proteína sobre la utilización del pasto estrella (Cynodon nlemfuensis) en pastoreo con ovinos. Zootecnia Trop. 19(Supl.1): 307-318.

Foidl, N; Mayorga, L; Vásquez, W. 1999. Utilización del Marango (Moringa oleifera) como forraje fresco para el ganado. Conferencia Electrónica de la FAO sobre Agroforestería para la Producción Animal en América Latina. htpp://www.fao.org/livestock/agap/frg/agrofor1/foidl16.htm.

Garza F, JD; Owens, FN; Welty, S. 1991. Effect of post-ruminal protein infusion on feed intake and utilization of low quality hay by beef steers. Miscellaneous publication Agric. Exp. Station, Oklahoma State Univ. USA. No. 134, 106-113.

Goodchild, AV; Mcmeniman, NP. 1994. Intake and digestibility of low quality roughages when supplemented with leguminous browse. J. Agric. Sci.122: 151-160.

Ibrahim, M; Franco, M; Pezo, DA; Camero, A; Araya, JL. 2001. Promoting intake of Cratylia argentea as a dry season supplement for cattle grazing Hyparrhenia rufa in the subhumid tropics. Agrofor. Syst. 51: 167-175.

Kass, M; Rodriguez, G. 1993. Evaluación Nutricional de Alimentos. CATIE, Turrialba, Costa Rica.

Ketelaars, 1991. Citado por el proyecto Biomasa, 1999. Guía técnica del cultivo de Marango (Moringa oleifera) Universidad Nacional de Ingeniería. Nicaragua. Cooperación Técnica de la Republica de Austria.

Kil, SJ; Froetschel, MA. 1994. Involvement of opioid peptides from casein on reticular motility and digesta passage in steers. J. Dairy Sci. 77: 111-123.

Leng, RA. 1990. Factors affecting the utilization of 'poor quality' forages by ruminants particularly under tropical conditions. Nutr. Res. Rev. 44: 277-303.

Makkar H, PS; Becker, K. 1996. Nutritional value and antinutritional components of whole and ethanol extracted Moringa oleifera leaves. Anim. Feed Sci. Technol. 63: 211-228.

Makkar H, PS; Becker, K. 1997. Nutrients and antiquality factors in different morphological parts of the Moringa oleifera tree. J. Agric. Sci., Cambridge 128: 311-332.

Malafaia, P; Silva, CL; Mendoca, RA; Magnoli, CR; Brandao, CA. 2003. Protein-energy supplementation for cattle raised on tropical pastures. Theoretical aspects and main results published in Brazil. Livest. Res. Rur. Dev.15:12. 
Medina, R; Sánchez, A. 2006. Efecto de la suplementación con follaje de Leucaena leucocephala sobre la ganancia de peso de ovinos desparasitados y no desparasitados contra estrongílidos digestivos. Zootecnia Tropical 24(1):55-68.

Mendieta, B. 1996. Administración Agropecuaria. Texto Básico. Universidad Nacional Agraria. Managua, Nicaragua.

Mendoza, A; Michailos, J; De Combellas, J. 2001. Uso de Leucaena en dietas completas para

corderos en crecimiento. Memorias III Congreso Nacional y I Internacional de Ovinos y Caprinos. Univ. Central de Venezuela. Maracay, estado Aragua. Venezuela.

MINITAB. 1998. Minitab User's Guide 2. Data Analysis and Quality tools, Release 12 for Windows, Windows 95 and Windows NT. Minitab Inc. Pennsylvania, USA.

Minson, DJ; Cowan, T; Havilah, E. 1993. Northern dairy feedbase 2001. Summer pasture and crops. Trop. Grassl. 27: 131-149.

Minson, DJ; Milford, R. 1967. The voluntary intake and digestibility of diets containing different proportion of legumes and mature pangola grass (Digitaria decumbens). Austr. J. Exp. Agric. Anim. Husb. 7:546-551.

Palma J, M; Huerta, A. 1999. Engorda de Ovinos en Confinamiento con Diferentes Niveles de Inclusión de Heno de Leucaena leucocephala. http://www.cipav.org.co/redagrofor/memorias99/P-Palma.htm

Perdomo, P. 1991. Adaptación edáfica y valor nutritivo de 25 especies y accesiones de leguminosas arbóreas y arbustivas en dos suelos contrastantes. Tesis de Zootecnia, Universidad Nacional de Colombia, Fac. de Ciencias Agrop. Palmira, Colombia. 128 p.

Pezo, D. 1991. Producción Ganadera en un Contexto Agroforestal. El Chasqui (CR) 25:1-2

Reyes Sánchez, Nadir; Ledin, Stig; Ledin, Inger. 2006. Biomass production and chemical composition of Moringa oleifera under different management regimes in Nicaragua. agroforestry systems 66:231-242

Reyes Sánchez, Nadir; Sporndly, Eva; Ledin, Inger. 2006. Effect of feeding different levels of foliage of Moringa oleifera to creole dairy cows on intake, digestibility, milk production and composition. Livestock Science 101: 24-31.

Ríos, LP; Rondón, MZ; Combellas, JB; Álvarez, ZR. 2005. Uso de morera (Morus sp.) y mata ratón (Gliricidia sepium) como sustitutos del alimento concentrado para corderos en crecimiento. Zootecnia Tropical 23(1): 49-60.

Vélez, M. 1993. Producción de cabras y ovejas en el trópico. Escuela Agrícola Panamericana (EAP), El Zamorano, Honduras. 